\title{
Schauder-Tychonoff Fixed-Point Theorem in Theory of Superconductivity
}

\author{
Mariusz Gil and Stanisław Wędrychowicz \\ Department of Mathematics, Rzeszów University of Technology, al.Powstańców Warszawy 6, 35-959 Rzeszów, Poland \\ Correspondence should be addressed to Stanisław Wędrychowicz; swedrych@prz.edu.pl
}

Received 25 April 2013; Accepted 5 June 2013

Academic Editor: Józef Banaś

Copyright ( 2013 M. Gil and S. Wędrychowicz. This is an open access article distributed under the Creative Commons Attribution License, which permits unrestricted use, distribution, and reproduction in any medium, provided the original work is properly cited.

\begin{abstract}
We study the existence of mild solutions to the time-dependent Ginzburg-Landau ((TDGL), for short) equations on an unbounded interval. The rapidity of the growth of those solutions is characterized. We investigate the local and global attractivity of solutions of TDGL equations and we describe their asymptotic behaviour. The TDGL equations model the state of a superconducting sample in a magnetic field near critical temperature. This paper is based on the theory of Banach space, Fréchet space, and Sobolew space.
\end{abstract}

\section{Introduction}

The objective of the paper is to investigate the existence and asymptotic behaviour of mild solutions on an unbounded interval of time-dependent Ginzburg-Landau equations (TDGL, for short) in superconductivity.

In the Ginzburg-Landau theory of phase transitions [1], the state of a superconducting material near the critical temperature is described by a complex-valued order parameter $\psi$, a real vector-valued vector potential $\mathbf{A}$, and, when the system changes with time, a real-valued scalar potential $\phi$. The latter is a diagnostic variable; $\psi$ and $\mathbf{A}$ are prognostic variables, whose evolution is governed by a system of coupled differential equations:

$$
\begin{gathered}
\eta\left(\frac{\partial}{\partial t}+i \kappa \phi\right) \psi=-\left(\frac{i}{\kappa} \nabla+\mathbf{A}\right)^{2} \psi+\left(1-|\psi|^{2}\right) \psi, \\
\frac{\partial \mathbf{A}}{\partial t}+\nabla \phi=-\nabla \times \nabla \times \mathbf{A}+\mathbf{J}_{s}+\nabla \times \mathbf{H} .
\end{gathered}
$$

The supercurrent density $\mathbf{J}_{s}$ is a nonlinear function of $\psi$ and A,

$$
\begin{aligned}
\mathbf{J}_{s} & \equiv \mathbf{J}_{s}(\psi, \mathbf{A})=\frac{1}{2 i \kappa}\left(\psi^{*} \nabla \psi-\psi \nabla \psi^{*}\right)-|\psi|^{2} \mathbf{A} \\
& =-\operatorname{Re}\left[\psi^{*}\left(\frac{i}{\kappa} \nabla+\mathbf{A}\right) \psi\right] .
\end{aligned}
$$

The system of (1)-(3) must be satisfied everywhere in $\Omega$, the region occupied by the superconducting material, and at all times $t>0$. The boundary conditions associated with the differential equations have the form

$$
\begin{gathered}
\mathbf{n} \cdot\left(\frac{i}{\kappa} \nabla+\mathbf{A}\right) \psi+\frac{i}{\kappa} \gamma \psi=0, \\
\mathbf{n} \times(\nabla \times \mathbf{A}-\mathbf{H})=\mathbf{0}
\end{gathered}
$$

on $\partial \Omega$, where $\partial \Omega$ is the boundary of $\Omega$ and $\mathbf{n}$ is the local outer unit normal to $\partial \Omega$. They must be satisfied at all times $t>0$.

We prove that the systems of (1)-(5) can be reduced to a semilinear equation; to use the appropriate theorem, we investigate the local and global attractivity of solutions of equations in question and describe their asymptotic behaviour.

In this paper, we consider the existence and asymptotic behaviour of mild solutions on an unbounded interval of the semilinear evolution equation of the following form:

$$
\begin{gathered}
\frac{d u}{d t}+\mathscr{A} u=\mathscr{F}(t, u(t)), \quad t \in \mathbb{R}_{+}=[0,+\infty), \\
u(0)=u_{0} \in \mathfrak{X},
\end{gathered}
$$

where the operator $\mathscr{A}: D(\mathscr{A}) \subset \mathfrak{X} \rightarrow \mathfrak{X}$ generates a $C_{0^{-}}$ semigroup $\left\{e^{-\mathscr{A}(t-s)}\right\}_{t \geq 0}$ and $\mathfrak{X}$ is a real Banach space.

Recently, a lot of papers have appeared that deal with the same or similar equations on a bounded interval (see [2-21]). 
However, only in a few papers, problem (6)-(7) was considered on an unbounded interval $[10,22]$. Additionally, in assumptions concerning the semigroup $\left\{e^{-\mathscr{A}(t-s)}\right\}_{t \geq 0}$ or the function $\mathscr{F}(t, u)$, rather restrictive conditions have been imposed which frequently require the compactness of $\mathscr{F}(t, u)$ or $\left\{e^{-\mathscr{A}(t-s)}\right\}_{t \geq 0}$ or equicontinuity of semigroup $\left\{e^{-\mathscr{A}(t-s)}\right\}_{t \geq 0}$ $[2-11,13-22]$. It is worthwhile mentioning that only a few papers have discussed asymptotic behaviour of solutions, mostly without the formulation of existence theorems $[10,23$, 24].

In this paper, we present conditions guaranteeing the existence of mild solutions on an unbounded interval of problem (6)-(7). We dispense with assumptions on the compactness of $\mathscr{F}(t, u)$ or $\left\{e^{-\mathscr{A}(t-s)}\right\}_{t \geq 0}$.

Moreover, we formulate theorems about asymptotic properties and both local and global attractivity of solutions of problem (6)-(7). The existence theorems concerning that problem will be proved with the help of the technique of a family of measures of noncompactness in the Fréchet space $C\left(\mathbb{R}_{+}, \mathfrak{X}\right)$ and Schauder-Tychonoff fixed-point principle.

The approach applied here was introduced and developed in [20, 25-35], for instance.

The paper is organized as follows. In Section 2, there are given notation and auxiliary facts that are needed further on. In Section 3, we formulate and prove a theorem on the existence of mild solution of (6) with condition (7). Moreover, the rate of the growth of those solutions is characterized.

Section 4 contains a theorem on local and global attractivity of solutions of problem (6)-(7). In Section 5, we give a theorem describing the asymptotic behaviour of solutions of (6)-(7).

Finally, in Section 6, we formulate the gauged TDGL equation as an abstract evolution equation in a Hilbert space. Moreover, this section is devoted to present examples of application of previously obtained theorems for TDGL equations.

In order to convert the systems equations (1)-(5) to the Cauchy problem (6)-(7) we are based on the papers and monographs (see $[1,30,36-69])$.

\section{Preliminaries}

Let $(\mathfrak{X},\|\cdot\|)$ be a real Banach space with the zero element $\theta$. Denote by $B(x, r)$ the closed ball in $\mathfrak{X}$ centered at $x$ and with radius $r$. If $X$ is a subset of a linear topological space, then the symbols $\bar{X}$ and Conv $X$ stand for the closure and the convex closure of $X$, respectively.

Let $\chi$ denote the Hausdorff measure of noncompactness in $\mathfrak{X}$, defined on bounded subsets $X$ of $\mathfrak{X}$ in the following way (see [27]):

$$
\chi(X):=\inf \{r>0: X \text { can be covered with finite }
$$

numbers of balls of radius $r$.

Further, denote by $C\left(\mathbb{R}_{+}, \mathfrak{X}\right)$ the Fréchet space consisting of all functions defined and continuous on $\mathbb{R}_{+}$with values in a Banach space $\mathfrak{X}$. The space $C\left(\mathbb{R}_{+}, \mathfrak{X}\right)$ is furnished with the family of seminorms;

$$
\begin{aligned}
& \|u\|_{n}:=\sup \{\|u(t)\|: t \in[0, n]\} \\
& \text { for } n=1,2, \ldots, u \in C\left(\mathbb{R}_{+}, \mathfrak{X}\right) .
\end{aligned}
$$

Let us recall two facts:

$\left(^{*}\right)$ a sequence $\left\{u_{n}\right\}_{n=1}^{\infty}$ is convergent to $u$ in $C\left(\mathbb{R}_{+}, \mathfrak{X}\right)$ if and only if $\left\{u_{n}\right\}_{n=1}^{\infty}$ is uniformly convergent to $x$ on compact subsets of $\mathbb{R}_{+}$;

$\left(^{* *}\right)$ a subset $U \subset C\left(\mathbb{R}_{+}, \mathfrak{X}\right)$ is relatively compact if and only if the restrictions to $[0, T]$ of all functions from $U$ form an equicontinuous set for each $T>0$ and $U(t)$ is relatively compact in $\mathfrak{X}$ for each $t \in \mathbb{R}_{+}$, where $U(t)=$ $\{u(t): u \in U\}$.

Moreover, we recall that a nonempty subset $U \subset$ $C\left(\mathbb{R}_{+}, \mathfrak{X}\right)$ is said to be bounded if

$$
\sup \left\{\|u\|_{n}: u \in U\right\}<\infty \text { for } n=1,2, \ldots
$$

Further, the family of all nonempty and bounded subsets of $C\left(\mathbb{R}_{+}, \mathfrak{X}\right)$ will be denoted by $\mathfrak{M}_{C}$, while the family of all nonempty and relatively compact subsets of $C\left(\mathbb{R}_{+}, \mathfrak{X}\right)$ is denoted by $\mathfrak{N}_{C}$. Obviously $\mathfrak{N}_{C} \subset \mathfrak{M}_{C}$.

We will use a family of measures of noncompactness $\left\{\mu_{T}\right\}_{T \geq 0}$ in the Fréchet space $C\left(\mathbb{R}_{+}, \mathfrak{X}\right)$ which was introduced in $[20,35]$. In order to define these measures, recall some quantities [25-27]. Let us fix a nonempty bounded subset $U$ of the space $C\left(\mathbb{R}_{+}, \mathfrak{X}\right)$. For $u \in U, \varepsilon \geq 0$, and $T \geq 0$ denote by $\omega^{T}(u, \varepsilon)$ the modulus of continuity of the function $u$ on the interval $[0, T]$, that is,

$$
\begin{aligned}
\omega^{T}(u, \varepsilon):=\sup \left\{\left\|u\left(t_{2}\right)-u\left(t_{1}\right)\right\|:\right. \\
\left.\qquad t_{1}, t_{2} \in[0, T],\left|t_{2}-t_{1}\right| \leq \varepsilon\right\} .
\end{aligned}
$$

Further, let us put

$$
\begin{gathered}
\omega^{T}(U, \varepsilon):=\sup \left\{\omega^{T}(u, \varepsilon): u \in U\right\}, \\
\omega_{0}^{T}(U):=\lim _{\varepsilon \rightarrow 0+} \omega^{T}(U, \varepsilon) .
\end{gathered}
$$

Obviously, the set $U$ is equicontinuous on $[0, T]$ if and only if $\omega_{0}^{T}(U)=0$.

Next, let us define the functions $\left\{\mu_{T}\right\}_{T \geq 0}$ on the family of bounded subsets of $C\left(\mathbb{R}_{+}, \mathfrak{X}\right)$ by the following formula:

$$
\mu_{T}(U):=\omega_{0}^{T}(U)+\sup \{\chi(U(t)): t \in[0, T]\} .
$$

It can be shown that the family $\left\{\mu_{T}\right\}_{T \geq 0}$ has the following properties:

$1^{o}$ the family $\operatorname{ker}\left\{\mu_{T}\right\}:=\left\{U \in \mathfrak{M}_{C}: \mu_{T}(U)=0\right.$ for $T \geq$ $0\}$ is nonempty and $\operatorname{ker}\left\{\mu_{T}\right\}=\mathfrak{N}_{C}$;

$$
\begin{aligned}
& 2^{o} U \subset V \Rightarrow \mu_{T}(U) \leq \mu_{T}(V) \text { for } T \geq 0 ; \\
& 3^{o} \mu_{T}(\mathrm{Conv} U)=\mu_{T}(U) \text { for } T \geq 0 ;
\end{aligned}
$$


$4^{o}$ if $\left\{U_{n}\right\}_{n=1}^{\infty}$ is a sequence of closed sets from $\mathfrak{M}_{C}$ such that $U_{n+1} \subset U_{n}(n=1,2, \ldots)$ and if $\lim _{n \rightarrow \infty} \mu_{T}\left(U_{n}\right)=$ 0 for each $T \geq 0$, then the intersection set $U_{\infty}:=$ $\bigcap_{n=1}^{\infty} U_{n}$ is nonempty.

Remark 1. Observe that in contrast to the definition of the concept of a measure of noncompactness given in [27], our mapping $\mu_{T}$ may take the value $\infty$. Moreover, a single mapping $\mu_{T}$ is not the measure of noncompactness in $C\left(\mathbb{R}_{+}, \mathfrak{X}\right)$ but the whole family $\left\{\mu_{T}\right\}_{T \geq 0}$ can be called family of measures of noncompactness.

Remark 2. Let us notice that the intersection set $U_{\infty}$ described in axiom $4^{\circ}$ is a member of the kernel of the family of measure of noncompactness $\left\{\mu_{T}\right\}_{T \geq 0}$, and therefore, $U_{\infty}$ is compact in $C\left(\mathbb{R}_{+}, \mathfrak{X}\right)$. In fact, the inequality $\mu_{T}\left(U_{\infty}\right) \leq$ $\mu_{T}\left(U_{n}\right)$ for $n=1,2, \ldots$ and $T \geq 0$ implies that $\mu_{T}\left(U_{\infty}\right)=0$. Hence, $U_{\infty} \in \operatorname{ker}\left\{\mu_{T}\right\}$. This property of the set $U_{\infty}$ will be very important in our further investigations.

Definition 3. A set $\left\{e^{-\mathscr{A} t}\right\}_{t \geq 0}$ of bounded linear operators on $\mathfrak{X}$ is called $C_{0}$-semigroup if

(i) $e^{-\mathscr{A} 0}=I, e^{-\mathscr{A}(t+s)}=e^{-\mathscr{A} t} e^{-\mathscr{A} s}$ for $t, s \geq 0$;

(ii) for all $x \in \mathfrak{X}$, the function $\mathbb{R}_{+} \ni t \mapsto e^{-\mathscr{A} t} x$ is continuous in $\mathbb{R}_{+}$.

Further, denote

$$
N(T):=\sup \left\{\left\|e^{-\mathscr{A} t}\right\|: t \leq T\right\} \quad \text { for } T \geq 0 .
$$

Definition 4. A function $x \in C\left(\mathbb{R}_{+}, \mathfrak{X}\right)$ is said to be a mild solution of the nonlocal initial-value problem (6)-(7) if for every $t \in \mathbb{R}_{+}$,

$$
u(t)=e^{-\mathscr{A} t} u_{0}+\int_{0}^{t} e^{-\mathscr{A}(t-s)} \mathscr{F}(s, u(s)) d s .
$$

To prove the existence results in this paper, we need the following lemmas.

Lemma 5 (see [21]). If $Y$ is a bounded subset of Banach space $\mathfrak{X}$, then for each $\mathcal{\varepsilon}>0$ there is a sequence $\left\{y_{k}\right\}_{k=1}^{\infty} \subset Y$ such that

$$
\chi(Y) \leq 2 \chi\left(\left\{y_{k}\right\}_{k=1}^{\infty}\right)+\varepsilon
$$

We call a set $U \subset L^{1}([0, T], \mathfrak{X})$ uniformly integrable if there exists $\eta \in L^{1}\left([0, T], \mathbb{R}_{+}\right)$such that $\|u(s)\| \leq \eta(s)$ for $u \in U$ and a.e. $s \in[0, T]$.

Lemma 6 (see [70]). If $\left\{u_{k}\right\}_{k=1}^{\infty} \subset L^{1}([0, T], \mathfrak{X})$ is uniformly integrable, then $\chi\left(\left\{u_{k}(t)\right\}_{k=1}^{\infty}\right)$ is measurable and

$$
\chi\left(\left\{\int_{0}^{t} u_{k}(s) d s\right\}_{k=1}^{\infty}\right) \leq 2 \int_{0}^{t} \chi\left(\left\{u_{k}(s)\right\}_{k=1}^{\infty}\right) d s \quad \text { for } t \in[0, T] .
$$

Lemma 7 (see [34]). Assume that a set $U \subset C\left(\mathbb{R}_{+}, \mathfrak{X}\right)$ is bounded. Then, (i) $\sup _{t \in[0, T]} \chi(U(t)) \leq \chi(U([0, T])) \leq \omega_{0}^{T}(U)+\sup _{t \in[0, T]}$ $\chi(U(t))$,

(ii) $\sup _{t \in[0, T]} \chi(U(t)) \leq \chi_{C}(U) \leq \omega_{0}^{T}(U)+\sup _{t \in[0, T]}$ $\chi(U(t))$,

for $T \geq 0$, where $U([0, T]):=\{u(t): t \in[0, T], u \in U\}$.

Lemma 8 (Gronwall's inequality [71]). Assume that the function $\phi_{1}: \mathbb{R}_{+} \rightarrow \mathbb{R}_{+}$is measurable and the function $\phi_{2}:$ $\mathbb{R}_{+} \rightarrow \mathbb{R}_{+}$is locally integrable. Moreover, we assume that $\int_{0}^{t} \phi_{1}(s) \phi_{2}(s) d s<\infty$ for $t \geq 0$ and a measurable function $\psi: \mathbb{R}_{+} \rightarrow \mathbb{R}_{+}$satisfies the following inequality:

$$
\psi(t) \leq \phi_{1}(t)+\int_{0}^{t} \phi_{2}(s) \psi(s) d s \quad \text { for } t \geq 0 .
$$

Then,

$$
\psi(t) \leq \phi_{1}(t)+\int_{0}^{t} \phi_{1}(s) \phi_{2}(s) \exp \left(\int_{s}^{t} \phi_{2}(\tau) d \tau\right) d s
$$

$$
\text { for } t \geq 0 \text {. }
$$

Next, we consider the operator $F: C\left(\mathbb{R}_{+}, \mathfrak{X}\right) \rightarrow$ $C\left(\mathbb{R}_{+}, \mathfrak{X}\right)$ defined by the following formula:

$$
\begin{array}{r}
(F u)(t):=\int_{0}^{t} e^{-\mathscr{A}(t-s)} \mathscr{F}(s, u(s)) d s, \quad t \in \mathbb{R}_{+}, \\
u \in C\left(\mathbb{R}_{+}, \mathfrak{X}\right) .
\end{array}
$$

Lemma 9 (see [20]). Assume that conditions $\left(H_{1}\right),\left(H_{2}\right)$, and $\left(H_{4}\right)$ are satisfied (see Section 3 ), a set $U \subset C\left(\mathbb{R}_{+}, \mathfrak{X}\right)$ is bounded, $T>0$, a function $h:[0, T] \rightarrow \mathbb{R}_{+}$is measurable, and

$$
2 N(T) \chi(\mathscr{F}(s, U(s))) \leq h(s)
$$

for a.e. $s \in[0, T]$. Then,

$$
\omega_{0}^{T}(F U) \leq \int_{0}^{T} h(s) d s .
$$

\section{Main Result}

In this section, we give an existence result for the semilinear equation of evolution (6)-(7), and we describe the asymptotic behaviour of those solutions.

First, we will assume that the functions involved in (6) satisfy the following conditions:

$\left(H_{1}\right) \mathscr{A}$ is a linear operator acting from $D(\mathscr{A}) \subset \mathfrak{X}$ and $\mathscr{A}$ generates $C_{0}$-semigroup $\left\{e^{-\mathscr{A}(t-s)}\right\}_{t \geq 0} ;$

$\left(H_{2}\right)$ the mapping $\mathscr{F}: \mathbb{R}_{+} \times \mathfrak{X} \rightarrow \mathfrak{X}$ satisfies the Carathéodory condition; that is, $\mathscr{F}(\cdot, u)$ is measurable for $u \in \mathfrak{X}$ and $\mathscr{F}(t, \cdot)$ is continuous for a.e. $t \in \mathbb{R}_{+}$;

$\left(H_{3}\right) a: \mathbb{R}_{+} \rightarrow \mathbb{R}_{+}$and $b: \mathbb{R}_{+} \rightarrow \mathbb{R}_{+}$are locally integrable functions such that

$$
\|\mathscr{F}(t, u)\| \leq a(t)+b(t)\|u\|
$$

for any $u \in \mathfrak{X}$ and a.e. $t \in \mathbb{R}_{+}$. 
$\left(H_{4}\right)$ There exists a locally integrable function $L: \mathbb{R}_{+} \rightarrow$ $\mathbb{R}_{+}$such that for any bounded $D \subset \mathfrak{X}$,

$$
\chi(\mathscr{F}(t, D)) \leq L(t) \chi(D)
$$

for a.e. $t \in \mathbb{R}_{+}$.

Theorem 10. Under assumptions $\left(H_{1}\right)-\left(H_{4}\right),(6)$ with initial condition (7) has, for every $u_{0} \in \mathfrak{X}$, at least one mild solution $u=u(t)$ which satisfied the following estimate:

$$
\begin{aligned}
\|u(t)\| \leq & M(t) \\
& +N(t) \int_{0}^{t} b(s) M(s) \exp \left(\int_{s}^{t} b(\tau) N(\tau) d \tau\right) d s
\end{aligned}
$$

where

$$
M(t)=N(t)\left(\left\|u_{0}\right\|+\int_{0}^{t} a(s) d s\right) .
$$

Proof. Consider the operator $G: C\left(\mathbb{R}_{+}, \mathfrak{X}\right) \rightarrow C\left(\mathbb{R}_{+}, \mathfrak{X}\right)$ defined by the following formula:

$$
G u(t):=e^{-\mathscr{A} t} u_{0}+\int_{0}^{t} e^{-\mathscr{A}(t-s)} \mathscr{F}(s, u(s)) d s .
$$

Now, let us observe that for any continuous function $u$ : $\mathbb{R}_{+} \rightarrow \mathfrak{X}$, in view of condition $\left(H_{3}\right)$, we get the following estimate:

$$
\|G u(t)\| \leq N(t)\left\|u_{0}\right\|+N(t) \int_{0}^{t}(a(s)+b(s)\|u(s)\|) d s,
$$

which yields

$$
\|G u(t)\| \leq M(t)+N(t) \int_{0}^{t} b(s)\|u(s)\| d s .
$$

Next, consider the following integral equation:

$$
\mathscr{P}(t)=M(t)+N(t) \int_{0}^{t} b(s) \mathscr{P}(s) d s
$$

Solving this equation by standard methods, we get

$$
\mathscr{P}(t)=M(t)+N(t) \int_{0}^{t} b(s) M(s) \exp \left(\int_{s}^{t} b(\tau) N(\tau) d \tau\right) d s .
$$

The function $\mathscr{P}(t)$ is continuous, nonnegative, and nondecreasing. Observe that the following implication is true:

$$
\|u(t)\| \leq \mathscr{P}(t) \Longrightarrow\|G u(t)\| \leq \mathscr{P}(t) \quad \text { for } t \geq 0 .
$$

Indeed, linking (29) and (32), we have

$$
\begin{aligned}
\|G u(t)\| & \leq M(t)+N(t) \int_{0}^{t} b(s)\|u(s)\| d s \\
& \leq M(t)+N(t) \int_{0}^{t} b(s) \mathscr{P}(s) d s=\mathscr{P}(t) .
\end{aligned}
$$

In the space $C\left(\mathbb{R}_{+}, \mathfrak{X}\right)$, let us consider the following set:

$$
\Gamma:=\left\{u \in C\left(\mathbb{R}_{+}, \mathfrak{X}\right):\|u(t)\| \leq \mathscr{P}(t), \text { for } t \geq 0\right\}
$$

Obviously, the set $\Gamma$ is convex and closed. Moreover, in view of (32), we have that $G$ is a self-mapping of $\Gamma$.

Using the criterion of convergence $\left({ }^{*}\right)$ in $C\left(\mathbb{R}_{+}, \mathfrak{X}\right)$ and standard techniques (see $[31-33,35]$ ), we can show that the operator $G$ is continuous on $C\left(\mathbb{R}_{+}, \mathfrak{X}\right)$.

Now, we consider the sequence of sets $\left\{\Gamma_{n}\right\}$ defined by induction as follows:

$$
\Gamma_{0}:=\Gamma, \quad \Gamma_{n+1}:=\operatorname{Conv}\left(G \Gamma_{n}\right) \quad \text { for } n=0,1, \ldots
$$

This sequence is decreasing, that is, $\Gamma_{n} \supset \Gamma_{n+1}$ for $n=$ $0,1,2 \ldots$.

Further, let us fix $T>0$ and for $t \in[0, T]$, let us put

$$
\begin{gathered}
g_{n}(t):=\sup \left\{\chi\left(\Gamma_{n}(s)\right): s \in[0, t]\right\} \\
w_{n}:=\omega_{0}^{T}\left(\Gamma_{n}\right) .
\end{gathered}
$$

The sequences $\left\{w_{n}\right\}$ and $\left\{g_{n}(t)\right\}$ are nonincreasing for all $t \in$ $[0, T]$, so they have limits

$$
\begin{aligned}
g_{\infty}(t):= & \lim _{n \rightarrow \infty} g_{n}(t), \quad t \in[0, T] \\
& w_{\infty}:=\lim _{n \rightarrow \infty} w_{n} .
\end{aligned}
$$

Moreover, each function $g_{n}$ is nondecreasing; therefore $g_{n}$, and $u_{\infty}$ are measurable on $[0, T]$ for $n=1,2, \ldots$.

Now, we apply the family of measures of noncompactness $\left\{\mu_{T}\right\}_{T \geq 0}$ defined in $C\left(\mathbb{R}_{+}, \mathfrak{X}\right)$ by formula (13). In view of the above notation, we have

$$
\mu_{T}\left(\Gamma_{n}\right)=w_{n}+g_{n}(T)
$$

We show that

$$
w_{\infty}=g_{\infty}(T)=0
$$

To fix a number $t \in[0, T], n \in \mathbb{N}$ and take an arbitrary number $s \in[0, t]$.

We know from Lemma 5 that for any $\varepsilon>0$, there is a sequence $\left\{y_{k}\right\}_{k=1}^{\infty} \subset G \Gamma_{n}(s)$, such that

$$
\chi\left(G \Gamma_{n}(s)\right) \leq 2 \chi\left(\left\{y_{k}\right\}_{k=1}^{\infty}\right)+\varepsilon .
$$

This implies that there is a sequence $\left\{u_{k}\right\}_{k=1}^{\infty} \subset \Gamma_{n}$, such that

$$
y_{k}=\left(G u_{k}\right)(s) \quad \text { for } k=1,2, \ldots
$$


Hence, in view of Lemma 6, $\left(H_{4}\right)$, and (36), we obtain

$$
\begin{aligned}
& \chi\left(G \Gamma_{n}(s)\right) \leq 2 \chi\left(\left\{y_{k}\right\}_{k=1}^{\infty}\right)+\varepsilon \\
& =2 \chi\left(\left\{\left(G u_{k}\right)(s)\right\}_{k=1}^{\infty}\right)+\varepsilon \leq 2 \chi\left(e^{-\mathscr{A} s} u_{0}\right) \\
& \quad+2 \chi\left(\left\{\int_{0}^{s} e^{-\mathscr{A}(s-\tau)} \mathscr{F}\left(\tau, u_{k}(\tau)\right) d \tau\right\}_{k=1}^{\infty}\right) \\
& \quad+\varepsilon \leq 4 \int_{0}^{s} \chi\left(e^{-\mathscr{A}(s-\tau)} \mathscr{F}\left(\tau,\left\{u_{k}(\tau)\right\}_{k=1}^{\infty}\right)\right) d \tau+\varepsilon \\
& \leq 4 N(T) \int_{0}^{s} L(\tau) \chi\left(\left\{u_{k}(\tau)\right\}_{k=1}^{\infty}\right) d \tau+\varepsilon \\
& \leq 4 N(T) \int_{0}^{t} L(\tau) g_{n}(\tau) d \tau+\varepsilon .
\end{aligned}
$$

Since $\varepsilon>0$ is arbitrary, it follows from the above inequalities that

$$
\chi\left(G \Gamma_{n}(s)\right) \leq 4 N(T) \int_{0}^{t} L(\tau) g_{n}(\tau) d \tau, \quad s \in[0, t]
$$

Using (36), we have

$$
\begin{aligned}
g_{n+1}(t) & =\sup \left\{\chi\left(G \Gamma_{n}(s)\right): s \in[0, t]\right\} \\
& \leq 4 N(T) \int_{0}^{t} L(\tau) g_{n}(\tau) d \tau, \quad t \in[0, T] .
\end{aligned}
$$

Letting $n \rightarrow \infty$, we derive the following inequality:

$$
g_{\infty}(t) \leq 4 N(T) \int_{0}^{t} L(\tau) g_{\infty}(\tau) d \tau, \quad t \in[0, T] .
$$

This inequality, together with Gronwall's Lemma 8, implies that

$$
g_{\infty}(t)=0, \quad t \in[0, T]
$$

Next let us notice that in view of $\left(H_{4}\right)$ and (36), we have

$$
\chi\left(\mathscr{F}\left(s, \Gamma_{n}(s)\right)\right) \leq L(s) \chi\left(\Gamma_{n}(s)\right) \leq L(s) g_{n}(s)
$$

for a.e. $s \in[0, T]$, and the function

$$
[0, T] \ni s \longmapsto 2 N(T) L(s) g_{n}(s)
$$

is measurable. Then, in virtue of equality $\omega_{0}^{T}\left(G \Gamma_{n}\right)=\omega_{0}^{T}\left(F \Gamma_{n}\right)$ and Lemma 9, we get

$$
\omega_{0}^{T}\left(G \Gamma_{n}\right) \leq 2 N(T) \int_{0}^{T} L(s) g_{n}(s) d s .
$$

Hence, we derive

$$
\begin{aligned}
w_{n+1} & =\omega_{0}^{T}\left(\Gamma_{n+1}\right)=\omega_{0}^{T}\left(G \Gamma_{n}\right) \\
& \leq 2 N(T) \int_{0}^{T} L(s) g_{n}(s) d s .
\end{aligned}
$$

Letting $n \rightarrow \infty$, we get

$$
w_{\infty} \leq 2 N(T) \int_{0}^{T} L(s) g_{\infty}(s) d s
$$

Keeping in mind (47), we deduce that

$$
w_{\infty}=0 \text {. }
$$

This together with (39) and (47) yields

$$
\lim _{n \rightarrow \infty} \mu_{T}\left(\Gamma_{n}\right)=\lim _{n \rightarrow \infty}\left(w_{n}+g_{n}(T)\right)=w_{\infty}+g_{\infty}=0 .
$$

Finally, using Remark 2 for the measure $\mu_{T}$, we deduce that the set $\Gamma_{\infty}:=\bigcap_{n=0}^{\infty} \Gamma_{n}$ is nonempty, convex, and compact. Then, by the Schauder-Tychonoff theorem, we conclude that operator $F: \Gamma_{\infty} \rightarrow \Gamma_{\infty}$ has at least one fixed-point $u=u(t)$. Obviously, the function $u=u(t)$ is a solution of problem (6)(7), and, in view of the definition of the set $\Gamma_{\infty}$, the estimate $\|u(t)\| \leq \mathscr{P}(t)$ holds to be true. This completes the proof.

\section{Local and Global Attractivity}

Following the concepts introduced in [36], we introduce first a few definitions of various kinds of the concept of attractivity of mild solution of (6).

Definition 11. The mild solution $u=u(t)$ of (6) with initial condition (7) is said to be globally attractive if for each mild solution $v=v(t)$ of (6) with initial condition $v(0)=v_{0}$ we have that

$$
\lim _{t \rightarrow \infty}(u(t)-v(t))=\theta
$$

In other words, we may say that solutions of (6) are globally attractive if for arbitrary solutions $u(t)$ and $v(t)$ of this equation condition (55) are satisfied.

Definition 12. We say that mild solution $u=u(t)$ of (6) with initial condition (7) is locally attractive if there exists a ball $B(u(0), r)$ in the space $\mathfrak{X}$ such that for arbitrary solution $v(t)$ of (1) with initial-value $v(0) \in B(u(0), r)$, condition (55) does hold.

In the case when the limit (55) is uniform with respect to all solutions $v(t)$, that is, when for each $\varepsilon>0$ there exist $T>0$ such that

$$
\|u(t)-v(t)\| \leq \varepsilon
$$

for all $v(t)$ being solutions of (6) with initial-value $v(0) \epsilon$ $B(u(0), r)$, and for $t \geq T$, we will say that solution $u=u(t)$ is uniformly locally attractive on $\mathbb{R}_{+}$.

Now, we formulate the main result of this section. We will consider (6) under the following conditions:

$\left(H_{1}^{\prime}\right) \mathscr{A}$ is the infinitesimal generator of an exponentially stable $C_{0}$-semigroup $\left\{e^{-\mathscr{A} t}\right\}_{t \geq 0}$; that is, there exist $M>$ $0, \rho>0$ such that $\left\|e^{-\mathscr{A} t}\right\| \leq M e^{-\rho t}$ for all $t \geq 0$; 
$\left(H_{5}\right)$ there exist locally integrable functions $m: \mathbb{R}_{+} \rightarrow \mathbb{R}_{+}$, such that

$$
\|\mathscr{F}(t, u)-\mathscr{F}(t, v)\| \leq m(t)\|u-v\|
$$

for $t \geq 0$ and $u, v \in \mathfrak{X}$. Moreover, we assume that

$$
\lim _{t \rightarrow \infty}\left(\int_{0}^{t} m(s) d s-\frac{\rho}{M} t\right)=-\infty
$$

Remark 13. The property $\left(H_{1}^{\prime}\right)$ is generally satisfied in diffusion problem. A necessary and sufficient condition for $\left(H_{1}^{\prime}\right)$ is presented in [72].

The main result of this section is shown in the given theorem below.

Theorem 14. Under assumptions $\left(H_{1}^{\prime}\right)$ and $\left(H_{2}\right)-\left(H_{5}\right)$, problem (6)-(7) has a mild solution $u=u(t)$ for each $u_{0} \in \mathfrak{X}$, which is globally attractive and locally uniformly attractive.

Proof. Existence of a solution $u=u(t)$ is a consequence of Theorem 10. Let us fix $r>0$ and $v_{0} \in B(u(0), r)$.

Let $v=v(t)$ denote a mild solution of (6) with the initial condition $v(0)=v_{0}$. Using $\left(H_{1}^{\prime}\right)$ and $\left(H_{3}\right)$, we get

$$
\begin{aligned}
\|v(t)\| \leq & \left\|e^{-\mathscr{A} t} v_{0}\right\|+\int_{0}^{t}\left\|e^{-\mathscr{A}(t-s)}\right\| \cdot\|\mathscr{F}(s, v(s))\| d s \\
\leq & M\left(\left\|u_{0}\right\|+r\right)+M \int_{0}^{t} e^{\rho s} a(s) d s \\
& +\int_{0}^{t} M e^{\rho s} b(s)\|v(s)\| d s .
\end{aligned}
$$

Now, let us put

$$
\begin{gathered}
\mathscr{P}_{1}(t)=M\left(\left\|v_{0}\right\|+r\right)+M \int_{0}^{t} e^{\rho s} a(s) d s, \\
\mathscr{P}_{2}(t)=M e^{\rho s} b(t) .
\end{gathered}
$$

Taking into account Lemma 5, we obtain

$$
\|v(t)\| \leq \mathscr{P}_{1}(t)+\int_{0}^{t} \mathscr{P}_{2}(s) \mathscr{P}_{1}(s) \exp \left(\int_{0}^{s} \mathscr{P}_{2}(\tau) d \tau\right) d s .
$$

Further, let $S$ be the set of all mild solutions $v(t)$ of (6) with the initial-value $v_{0}=v(0) \in B(u(0), r)$.

Put

$$
g(t)=\sup \{\|u(t)-v(t)\|: v \in S\} .
$$

The estimate (61) implies that the function $g(t)$ is well defined. Applying $\left(H_{5}\right)$ and $\left(H_{1}^{\prime}\right)$, we get

$$
\begin{aligned}
\|u(t)-v(t)\| \leq & \left\|e^{-\mathscr{A} t}\right\| \cdot\left\|u_{0}-v_{0}\right\| \\
& +\int_{0}^{t}\left\|e^{-\mathscr{A}(t-s)}\right\| m(s)\|u(s)-v(s)\| d s \\
\leq & M e^{-\rho t} r+\int_{0}^{t} M e^{-g(t-s)} m(s) g(s) d s,
\end{aligned}
$$

or, equivalently,

$$
e^{\rho t} g(t) \leq M r+\int_{0}^{t} M m(s) e^{\rho s} g(s) d s
$$

Using again Lemma 5 for the above estimate (where $h(t)=$ $\left.e^{\rho t} g(t)\right)$, we obtain

$$
\begin{aligned}
e^{\rho t} g(t) \leq & M r \\
& +\int_{0}^{t} M^{2} r m(s) \exp \left(\int_{s}^{t} M m(\tau) d \tau\right) d s .
\end{aligned}
$$

Elementary calculations lead to the following equality:

$$
\begin{gathered}
\int_{0}^{t} M^{2} r m(s) \exp \left(\int_{s}^{t} M m(\tau) d \tau\right) d s \\
\quad=M r\left(\exp \left(\int_{0}^{t} M m(s) d s\right)-1\right) .
\end{gathered}
$$

Hence,

$$
\begin{aligned}
g(t) \leq & M r \exp (-\rho t)+M r \exp \left(\int_{0}^{t} M m(s) d s-\rho t\right) \\
& -M r \exp (-\rho t) .
\end{aligned}
$$

Applying assumption $\left(H_{5}\right)$, we derive

$$
\lim _{t \rightarrow \infty} g(t)=0,
$$

and this proves that $u(t)$ is locally attractive. Finally, this equality together with definition of the function $g(t)$ implies that $u(t)$ is globally attractive. The proof is complete.

Remark 15. In the case when $m(t) \equiv m$ is constant, the following condition

$$
\lim _{t \rightarrow \infty}\left(\int_{0}^{t} m(s) d s-\frac{\rho}{M} t\right)=-\infty
$$

means that $m<\rho M$. Observe that this condition cannot be weakened. This observation is illustrated by the following exampled.

Example 16. Let $\mathfrak{X}=\mathbb{R}, m(t) \equiv m, \mathscr{F}(t, u)=\sin t+m u$, $M=1$, and $e^{-\mathscr{A} t}=e^{-\rho t}$. Then the equation

$$
u(t)=e^{-\mathscr{A} t} u_{0}+\int_{0}^{t} e^{-\mathscr{A}(t-s)} \mathscr{F}(s, u(s)) d s
$$

(for any fixed $u_{0} \in \mathbb{R}$ ) has the solution $u(t)$ expressed by the following formula:

$$
u(t)=\frac{(\rho-m) \sin t-\cos t}{(\rho-m)^{2}+1}+\left(u_{0}+\frac{1}{(\rho-m)^{2}+1}\right) e^{(m-\rho) t}
$$

Notice that for $m \geq \rho$, the solution $u(t)$ is neither globally attractive nor locally uniformly attractive, because for each 
other solution $v(t)$ with initial condition $v(0)=v_{0}$, obviously described by similar formula as $u(t)$, we would have a contradiction:

$$
|u(t)-v(t)|=\left\{\begin{array}{l}
\left|u_{0}-v_{0}\right| e^{(m-\rho) t} \longrightarrow \infty \\
\text { or } \\
\left|u_{0}-v_{0}\right| .
\end{array}\right.
$$

\section{Asymptotic Behaviour}

In this section, we will give a theorem describing asymptotic behaviour of mild solutions of (6) with condition (7). This theorem generalizes the result included in [72, Theorem 4.4]. First, we formulate the assumptions.

$\left(H_{3}^{\prime}\right)$ This condition is almost identical with $\left(H_{3}\right)$ and the only difference is that we assume the functions $a$ and $b$ are locally essentially bounded on $\mathbb{R}_{+}$.

$\left(H_{5}^{\prime}\right)$ There exists $u_{\infty} \in \mathfrak{X}$ such that there exists the limit $\lim _{t \rightarrow \infty} \mathscr{F}\left(t, u_{\infty}\right)$ and

$$
\lim _{t \rightarrow \infty} \mathscr{F}\left(t, u_{\infty}\right)=\mathscr{A}^{-1} u_{\infty} .
$$

Moreover, there exists a number $m<\rho / M$ such that

$$
\left\|\mathscr{F}(t, u)-\mathscr{F}\left(t, u_{\infty}\right)\right\| \leq m\left\|u-u_{\infty}\right\|
$$

for $t \geq 0$ and $u \in \mathfrak{X}$.

Remark 17. The condition $\left(H_{5}^{\prime}\right)$ in conjunction with $\left(H_{1}^{\prime}\right)$ ensures the existence of $\mathscr{A}^{-1}$ (see [72]). Clearly, $\left(H_{3}^{\prime}\right)$ implies $\left(H_{3}\right)$.

Theorem 18. Under assumptions $\left(H_{1}^{\prime}\right),\left(H_{2}\right),\left(H_{3}^{\prime}\right),\left(H_{4}\right)$, and $\left(H_{5}^{\prime}\right)$, (6) with condition (7) has a mild solution $u=u(t)$ for each $u_{0} \in \mathfrak{X}$ such that $\lim _{t \rightarrow \infty} u(t)=u_{\infty}$.

Proof. The existence of a mild solution $u(t)$ is guaranteed by Theorem 10. Let us put

$$
\begin{gathered}
g(t)=\left\|u(t)-u_{\infty}\right\|, \\
\mathscr{F}_{\infty}=\lim _{t \rightarrow \infty} \mathscr{F}\left(t, u_{\infty}\right) .
\end{gathered}
$$

We show that

$$
\lim _{t \rightarrow \infty} g(t)=0 .
$$

Recall that if assumption $\left(H_{1}^{\prime}\right)$ is fulfilled, then for each $z \in \mathfrak{X}$ we have (see [72])

$$
\int_{0}^{\infty} e^{-\mathscr{A} s} z d s=-\mathscr{A}^{-1} z
$$

Using the above fact and $\left(H_{5}^{\prime}\right)$, we get

$$
\begin{aligned}
u_{\infty} & =\left(-\mathscr{A}^{-1}\left(-\mathscr{A}^{-1} u_{\infty}\right)\right)=-\mathscr{A}^{-1} \mathscr{F}_{\infty} \\
& =\int_{0}^{\infty} e^{-\mathscr{A} s} \mathscr{F}_{\infty} d s .
\end{aligned}
$$

Linking the above equality with $\left(H_{1}^{\prime}\right)$, we obtain $g(t)$

$$
\begin{aligned}
& =\left\|e^{-\mathscr{A} t} u_{0}+\int_{0}^{t} e^{-\mathscr{A}(t-s)} \mathscr{F}(s, u(s)) d s-\int_{0}^{\infty} e^{-\mathscr{A} s} \mathscr{F}_{\infty} d s\right\| \\
& \leq M e^{-\rho t}\left\|u_{0}\right\|+\left\|\int_{t}^{\infty} e^{-\mathscr{A} s} \mathscr{F}_{\infty} d s\right\| \\
& +\left\|\int_{0}^{t} e^{-\mathscr{A}(t-s)} \mathscr{F}(s, u(s)) d s-\int_{0}^{t} e^{-\mathscr{A} s} \mathscr{F}_{\infty} d s\right\| \\
& \leq M e^{-\rho t}\left\|u_{0}\right\|+\frac{M}{e^{-\rho t} \rho\left\|\mathscr{F}_{\infty}\right\|} \\
& +\left\|\int_{0}^{t} e^{-\mathscr{A}(t-s)} \mathscr{F}(s, u(s)) d s-\int_{0}^{t} e^{-\mathscr{A}(t-s)} \mathscr{F}_{\infty} d s\right\| \\
& \leq M e^{-\rho t}\left(\left\|u_{0}\right\|+\frac{\left\|\mathscr{F}_{\infty}\right\|}{\rho}\right) \\
& +\int_{0}^{t} M e^{-\rho(t-s)}\left\|\mathscr{F}(s, u(s))-\mathscr{F}_{\infty}\right\| d s \\
& \leq M e^{-\rho t}\left(\left\|u_{0}\right\|+\frac{\left\|\mathscr{F}_{\infty}\right\|}{\rho}\right) \\
& +M e^{-\rho t} \int_{0}^{t} e^{\rho s}\left\|\mathscr{F}\left(s, u_{\infty}\right)-\mathscr{F}_{\infty}\right\| d s \\
& +M e^{-\rho t} \int_{0}^{t} e^{\rho s}\left\|\mathscr{F}(s, u(s))-\mathscr{F}\left(s, u_{\infty}\right)\right\| d s .
\end{aligned}
$$

Next, putting

$$
\begin{aligned}
c(t)= & M e^{-\rho(t)}\left(\left\|u_{0}\right\|+\frac{\left\|\mathscr{F}_{\infty}\right\|}{\rho}\right) \\
& +M e^{-\rho t} \int_{0}^{t} e^{\rho s}\left\|\mathscr{F}\left(s, u_{\infty}\right)-\mathscr{F}_{\infty}\right\| d s
\end{aligned}
$$

and applying $\left(H_{5}^{\prime}\right)$, we derive the following inequality:

$$
g(t) \leq c(t)+m M e^{-\rho t} \int_{0}^{t} e^{\rho s} g(s) d s .
$$

Hence,

$$
e^{\rho t} g(t) \leq e^{\rho(t)} c(t)+m M \int_{0}^{t} e^{\rho s} g(s) d s .
$$

The above inequality in conjunction with Lemma 5 gives

$$
\begin{aligned}
e^{\rho t} g(t) \leq & e^{\rho t} c(t) \\
& +\int_{0}^{t} e^{\rho s} c(s) m M \cdot \exp (m M(t-s)) d s \\
= & e^{\rho t} c(t) \\
& +m M e^{m M t} \int_{0}^{t} c(s) \exp ((\rho-m M) s) d s .
\end{aligned}
$$


Hence,

$$
g(t) \leq c(t)+\frac{m M \int_{0}^{t} c(s) e^{(g-m M) s} d s}{\exp ((\rho-m M) t)}
$$

Before proving (76), we first show that

$$
\lim _{t \rightarrow \infty} c(t)=0 .
$$

To prove this equality, it is sufficient to show that the last component in the formula expressing $c(t)$, that is, $M e^{-\rho t}$ $\int_{0}^{t} e^{\rho s}\left\|\mathscr{F}\left(s, u_{\infty}\right)-\mathscr{F}_{\infty}\right\| d s$, tends to zero as $t \rightarrow \infty$.

To this end, let us fix $\varepsilon>0$. Assumption $\left(H_{5}^{\prime}\right)$ implies that there exists $t_{0}$ such that

$$
\sup \left\{\left\|\mathscr{F}\left(s, u_{\infty}\right)-\mathscr{F}_{\infty}\right\|: s \geq t_{0}\right\} \leq \frac{\varepsilon \rho}{2 M} .
$$

This inequality together with $\left(H_{3}^{\prime}\right)$ implies

$$
\text { ess } \sup \left\{\left\|\mathscr{F}\left(s, u_{\infty}\right)-\mathscr{F}_{\infty}\right\|: s \in \mathbb{R}\right\}<\infty .
$$

Now, fix $t_{1} \in \mathbb{R}$ such that $t_{1} \geq t$ and

$$
\begin{aligned}
& \text { ess } \sup \left\{\left\|\mathscr{F}\left(s, u_{\infty}\right)-\mathscr{F}_{\infty}\right\|: s \in \mathbb{R}\right\} \\
& \leq \frac{\varepsilon \rho \exp \left(\rho t_{1}\right)}{2 M\left(\exp \left(\rho t_{0}\right)-1\right)} .
\end{aligned}
$$

Then using (86) and (88), we conclude that for $t \geq t_{1}$ we have

$$
\begin{aligned}
M e^{-\rho t} & \int_{0}^{t} e^{\rho s}\left\|\mathscr{F}\left(s, u_{\infty}\right)-\mathscr{F}_{\infty}\right\| d s \\
\leq & M e^{-\rho t} \int_{0}^{t_{0}} e^{\rho s}\left\|\mathscr{F}\left(s, u_{\infty}\right)-\mathscr{F}_{\infty}\right\| d s \\
& \quad+M e^{-\rho t} \int_{t_{0}}^{t} e^{\rho s}\left\|\mathscr{F}\left(s, u_{\infty}\right)-\mathscr{F}_{\infty}\right\| d s \leq \frac{\varepsilon}{2}+\frac{\varepsilon}{2}=\varepsilon .
\end{aligned}
$$

This fact proves (85).

Further, using $\left(H_{5}^{\prime}\right)$ and (85) and employing de L'Hospital's rule for the fraction on the right-hand side of inequality (84), we obtain that condition (76) is satisfied. This fact completes the proof.

\section{An Application to the Ginzburg-Landau Equations of Superconductivity}

In this section, we formulate the gauged time-dependent Ginzburg-Landau (TDGL) equations as an abstract evolution equation in a Hilbert space. Moreover, we show applications of the above theorems to TDGL equations.

We assume that $\Omega$ is a bounded domain in $\mathbb{R}^{n}$ with boundary $\partial \Omega$ of class $C^{1,1}$. That is, $\Omega$ is an open and connected set whose boundary $\partial \Omega$ is a compact $(n-1)$-manifold described by Lipschitz continuous differentiable charts. We consider two- and three-dimensional problems ( $n=2$ and $n=3$, resp.). Assume that the vector potential $\mathbf{A}$ takes its values in $\mathbb{R}^{n}$. The vector $\mathbf{H}$ will represent the (externally) applied magnetic field, which is a function of space and time; similarly to $\mathbf{A}$, it takes its values in $\mathbb{R}^{n}$. The function $\gamma$ is defined and satisfies Lipschitz condition on $\partial \Omega$, and $\gamma(x) \geq 0$ for $x \in \partial \Omega$. The parameters in the TDGL equations are $\eta$, a (dimensionless) friction coefficient, and $\kappa$, the (dimensionless) Ginzburg-Landau parameter.

The order parameter should be thought of as the wave function of the center-of-mass motion of the "superelectrons" (Cooper pairs), whose density is $n_{s}=|\psi|^{2}$ and whose flux is $\mathbf{J}_{s}$. The vector potential A determines the electromagnetic field; $\mathbf{E}=-\partial_{t} \mathbf{A}-\nabla \phi$ is the electric field and $\mathbf{B}=\nabla \times \mathbf{A}$ is the magnetic induction, where $\mathbf{J}$, the total current, is the sum of a "normal" current $\mathbf{J}_{n}=\mathbf{E}$, the supercurrent $\mathbf{J}_{s}$, and the transport current $\mathbf{J}_{t}=\nabla \times \mathbf{H}$. The normal current obeys Ohm's law $\mathbf{J}_{n}=\sigma_{n} \mathbf{E}$; the "normal conductivity" coefficient $\sigma_{n}$ is equal to one in the adopted system of units. The difference $\mathbf{M}=\mathbf{B}-\mathbf{H}$ is known as the magnetization. The trivial solution $(\psi=0, \mathbf{B}=\mathbf{H}, \mathbf{E}=\mathbf{0})$ represents the normal state, where all superconducting properties have been lost.

Now we accept the following notion: all Banach spaces are real; the (real) dual of a Banach space $X$ is denoted by $X^{\prime}$. The symbol $L^{p}(\Omega)$, for $1 \leq p \leq \infty$, denotes the usual Lebesgue space, with norm $\|\cdot\|_{L^{p}} ;(\cdot, \cdot)$ is the inner product in $L^{2}(\Omega)$. $W^{m, 2}(\Omega)$, for nonnegative integer $m$, is the usual Sobolev space, with norm $\|\cdot\|_{W^{m, 2}} ; W^{m, 2}(\Omega)$ is a Hilbert space for the inner product $(\cdot, \cdot)_{m, 2}$ given by $(u, v)_{m, 2}=\sum_{|\alpha| \leq m}\left(\partial^{\alpha} u, \partial^{\alpha} v\right)$ for $u, v \in W^{m, 2}(\Omega)$. Fractional Sobolev space $W^{s, 2}(\Omega)$, with a fractional $s$, is defined by interpolation ([40, Chap. VII], and $[41,49,50]) . C^{\nu}(\Omega)$, for $v \geq 0, v=m+\lambda$ with $0 \leq \lambda<1$, is the space of $m$ times continuous differentiable functions on $\Omega$; those $m$ th-order derivatives satisfy the Hölder condition with exponent $\lambda$ if $\nu$ is a proper fraction; the norm $\|\cdot\|_{C^{v}}$ is defined in the usual way.

The definitions extend to the space of vector-valued functions in the standard way, with the caveat that the inner product in $\left[L^{2}(\Omega)\right]^{n}$ is defined by $(u, v)=\int_{\Omega} u \cdot v$, where the symbol $(u, v)$ indicates the scalar product in $\mathbb{R}^{n}$. Complexvalued functions are interpreted as vector-valued functions with two real components.

Functions that vary in space and time, like the order parameter and the vector potential, are considered as mappings from the time domain, which is a subinterval of $[0, \infty)$, into spaces of complex- or vector-valued functions defined in $\Omega$. Let $X=\left(X,\|\cdot\|_{X}\right)$ be a Banach space of functions defined in $\Omega$. Then, the functions are defined in $\Omega$. Then, the functions of space and time defined on $\Omega \times(0, T)$, for $T>0$, may be considered as elements of $L^{p}(0, T ; X)$, for $1 \leq p \leq \infty$, or $W^{2, m}(0, T ; X)$, for nonnegative $m$, or $C^{\nu}(0, T ; X)$, for $\nu \geq 0$, $\nu=m+\lambda$ with $0 \leq \lambda<1$. Detailed definitions can be found, for example, in [43].

Obviously, function spaces of ordered pairs $(\psi, \mathbf{A})$, where $\psi: \Omega \rightarrow \mathbb{R}^{2}$ and $\mathbf{A}: \Omega \rightarrow \mathbb{R}^{n}(n=2,3)$, play an important role in the study of the gauged TDGL equations. We therefore adapt the following special notation: $\mathscr{H}=[X(\Omega)]^{2} \times[X(\Omega)]^{n}$, for any Banach space for the order parameter $\psi$ and the vector potential A, respectively. A suitable framework for the functional analysis of the gauged TDGL equations is the 
Cartesian product $\mathscr{W}^{1+\alpha, 2}=\left[W^{1+\alpha, 2}(\Omega)\right]^{2} \times\left[W^{1+\alpha, 2}(\Omega)\right]^{n}$, where $\alpha \in(1 / 2,1)$. This space is continuously imbedded in $\mathscr{W}^{1,2} \cap \mathscr{L}^{\infty}$.

Assume $\mathbf{H} \neq \mathbf{0}$ and $\mathbf{H} \in\left[L^{2}(\Omega)\right]^{n}$. Let $\mathbf{A}_{\mathbf{H}}$ be a minimizer of the convex quadratic form $J_{\omega} \equiv \mathbf{J}_{\omega}[A]$,

$$
J_{\omega}[\mathbf{A}]=\int_{\Omega}\left[\omega(\nabla \cdot \mathbf{A})^{2}+|\nabla \times \mathbf{A}-\mathbf{H}|^{2}\right] d x,
$$

on the domain

$$
\mathscr{D}\left(J_{\omega}\right)=\left\{\mathbf{A} \in\left[W^{1,2}(\Omega)\right]^{n}: \mathbf{n} \cdot \mathbf{A}=0 \text { on } \partial \Omega\right\} .
$$

We now introduce the reduced vector potential $\mathbf{A}^{\prime}$ :

$$
\mathbf{A}^{\prime}=\mathbf{A}-\mathbf{A}_{\mathbf{H}} .
$$

In terms of $\psi$ and $\mathbf{A}^{\prime}$ the gauged TDGL equations have the following form:

$$
\begin{gathered}
\frac{\partial \psi}{\partial t}-\frac{1}{\eta \kappa^{2}} \Delta \psi=\varphi \quad \text { in } \Omega \times(0, \infty) \\
\frac{\partial \mathbf{A}^{\prime}}{\partial t}+\nabla \times \nabla \times \mathbf{A}^{\prime}-\omega \nabla\left(\nabla \cdot \mathbf{A}^{\prime}\right)=\mathbf{F} \quad \text { in } \Omega \times(0, \infty) \\
\mathbf{n} \cdot \nabla+\gamma \psi=0, \quad \mathbf{n} \cdot \mathbf{A}^{\prime}=0, \\
\mathbf{n} \times\left(\nabla \cdot \mathbf{A}^{\prime}\right)=\mathbf{0} \quad \text { on } \partial \Omega \times(0, \infty) .
\end{gathered}
$$

Here, $\varphi$ and $\mathbf{F}$ are nonlinear functions of $\psi$ and $\mathbf{A}^{\prime}$ :

$$
\begin{gathered}
\varphi \equiv \varphi\left(t, \psi, \mathbf{A}^{\prime}\right)=\frac{1}{\eta}\left[-\frac{2 i}{\kappa}(\nabla \psi) \cdot\left(\mathbf{A}^{\prime}+\mathbf{A}_{\mathbf{H}}\right)\right. \\
-\frac{i}{\kappa}\left(1-\eta \kappa^{2} \omega\right) \psi\left(\nabla \cdot \mathbf{A}^{\prime}\right) \\
\left.-\psi\left|\mathbf{A}^{\prime}+\mathbf{A}_{\mathbf{H}}\right|^{2}+\left(1-|\psi|^{2}\right) \psi\right] \\
\mathbf{F} \equiv \mathbf{F}\left(t, \psi, \mathbf{A}^{\prime}\right)=\frac{1}{2 i k}\left(\psi^{*} \nabla \psi-\psi \nabla \psi^{*}\right) \\
-|\psi|^{2}\left(\mathbf{A}^{\prime}+\mathbf{A}_{\mathbf{H}}\right)-\frac{\partial \mathbf{A}_{\mathbf{H}}}{\partial t}
\end{gathered}
$$

The equations are supplemented by initial data, which is in the followimg form:

$$
\psi=\psi_{0}, \quad \mathbf{A}=\mathbf{A}_{0} \quad \text { on } \Omega \times\{0\},
$$

where $\psi_{0}$ and $\mathbf{A}_{0}$ are given, and by (92), we have

$$
\psi=\psi_{0}, \quad \mathbf{A}^{\prime}=\mathbf{A}_{0}-\mathbf{A}_{\mathbf{H}}(0) \quad \text { on } \Omega \times\{0\} .
$$

In the next part we connect the evolution of the solution $\left(\psi, \mathbf{A}^{\prime}\right)$ of the system of (93) with the initial data $\left(\psi_{0}, \mathbf{A}_{0}-\right.$ $\left.\mathbf{A}_{\mathbf{H}}(0)\right)$ with the dynamics of a vector $u$ in a Hilbert, space

$$
\mathscr{L}^{2}=\left[L^{p}(\Omega)\right]^{2} \times\left[L^{p}(\Omega)\right]^{n} .
$$

The following analysis is restricted to the case $\omega>0$ and the case $\omega=0$ (see [73]).

Let vector $u:[0, \infty) \rightarrow \mathscr{L}^{2}$ represent the pair $\left(\psi, \mathbf{A}^{\prime}\right)$,

$$
u=\left(\psi, \mathbf{A}^{\prime}\right) \equiv\left(\psi, \mathbf{A}-\mathbf{A}_{\mathbf{H}}\right)
$$

and let $\mathscr{A}$ be the linear self-adjoint operator in $\mathscr{L}^{2}$ associated with the quadratic form $Q_{\omega} \equiv Q_{\omega}[u]$ given by the following formula:

$$
\begin{aligned}
Q_{\omega}[u]= & \int_{\Omega}\left[\frac{1}{\eta \kappa^{2}}|\nabla \psi|^{2}+\omega\left(\nabla \cdot \mathbf{A}^{\prime}\right)^{2}+\left|\nabla \times \mathbf{A}^{\prime}\right|^{2}\right] d x \\
& +\int_{\partial \Omega} \frac{\gamma}{\eta \kappa^{2}}|\psi|^{2} d \sigma(x),
\end{aligned}
$$

on the domain

$$
\begin{aligned}
\mathscr{D}\left(Q_{\omega}\right) & =\mathscr{D}\left(\mathscr{A}^{1 / 2}\right) \\
& =\left\{u=\left(\psi, \mathbf{A}^{\prime}\right) \in \mathscr{W}^{1,2}: \mathbf{n} \cdot \mathbf{A}^{\prime}=0 \text { on } \partial \Omega\right\} .
\end{aligned}
$$

The quadratic form $Q_{\omega}$ is nonnegative. Furthermore, since $\omega>0, Q_{\omega}\left[\psi, \mathbf{A}^{\prime}\right]+c\|\psi\|_{L^{2}}$ is coercive on $\mathscr{W}^{1,2}$ for any constant $c>0$. Hence, $\mathscr{A}$ is positively definite in $\mathscr{L}^{2}$ ([44, Chap. 1], equation $(5,45))$. If it does not lead to confusion, we use the same symbol $\mathscr{A}$ for the restriction $\mathscr{A}_{\psi}$ and $\mathscr{A}_{\mathrm{A}}$ of $\mathscr{A}$ to the respective linear subspace $\left[L^{2}(\Omega)\right]^{2}=\left[L^{2}(\Omega)\right]^{2} \times\{0\}($ for $\psi)$ and $\left[L^{2}(\Omega)\right]^{n} \equiv\{0\} \times\left[L^{2}(\Omega)\right]^{n}$ (for $\left.\mathbf{A}\right)$ of $\mathscr{L}^{2}$.

Now, consider the initial-value problems (6) and (7) in $\mathscr{L}^{2}$, where $\mathscr{F}(t, u)=(\varphi, \mathbf{F}), \varphi$ and $\mathbf{F}$ is given by (94) and (95) and $u_{0}=\left(\psi_{0}, \mathbf{A}_{0}-\mathbf{A}_{\mathbf{H}}(0)\right)$.

With $\lambda \in(1 / 2,1)$ and $u_{0} \in \mathscr{W}^{1+\lambda, 2}$, we say that $u$ are a solution of (6) and (7) on the interval $[0, T]$, for some $T>0$, if $u:[0, T] \rightarrow \mathscr{W}^{1+\lambda, 2}$ is continuous and

$$
u(t)=e^{-\mathscr{A}(t)}+\int_{0}^{t} e^{-\mathscr{A}(t-s)} \mathscr{F}(s, u(s)) d s \quad \text { for } t \in[0, T],
$$

in $\mathscr{L}^{2}$. A mild solution of the initial-value problems (6) and (7) defines a weak solution $\left(\psi, \mathbf{A}^{\prime}\right)$ of the boundary value problem (93), which in turn defines a weak solution $(\psi, \mathbf{A})$ of the gauged TDGL equations, provided $\mathbf{A}_{\mathbf{H}}$ is sufficiently regular.

Namely, let us assume that $\mathfrak{X}=\mathscr{L}^{2}$ and $u_{0} \in \mathscr{L}^{2}$, and $u: \mathbb{R}_{+} \times \mathbb{R}^{n} \rightarrow \mathbb{R}^{n}$ for $n \in \mathbb{N}$ is an unknown function, $u=$ $u(t, x)$. In order to apply Theorems 10 and 14 , we are not going to consider $u$ as a function of $t$ and $x$ together, but rather as a mapping $u$ of variable $t$ into the space $\mathfrak{X}=\mathscr{L}^{2}$ of functions $x$, that is, $u: \mathbb{R}_{+} \rightarrow \mathscr{L}^{2}, u(t)(x)=u(t, x), x \in \mathbb{R}^{n}, t \in \mathbb{R}_{+}$.

Remark 19. Since $\mathscr{A}$ is the linear self-adjoint operator in $\mathscr{L}^{2}$ associated with the quadratic form (100), then $\mathscr{A}$ generates a semigroup $\left\{e^{-\mathscr{A} t}\right\}_{t \geq 0}$ (see [74]).

Below, we formulate the principal theorem of this paper. This theorem is a simple consequence of Theorem 10. 
Theorem 20. Under assumptions $\left(H_{1}\right)-\left(H_{4}\right)$, problem (6)-(7) is equivalent to the system of (1)-(5) (TDGL equations) and has for each $u_{0} \in \mathscr{L}^{2}$ at least one mild solution in sense of Definition $4 u=u(t)$. Moreover, each solution $u(t)$ satisfies estimate (25).

We observe that in the case when $\left\{e^{-\mathscr{A t}}\right\}_{t \geq 0}$ is the semigroup of contractions and $a(t) \equiv a, b(t) \equiv b$ for $t \epsilon$ $\mathbb{R}_{+}$are constant, after simple calculations based on estimate (25), we get that the solution $u=u(t)$ has the asymptotic characterization:

$$
\|u(t)\|=O\left(t^{3}\right), \quad \text { as } t \longrightarrow \infty .
$$

For further purposes, let us formulate the following assumption:

(A) $\mathscr{A}$ is the infinitesimal generator of an exponentially stable $C_{0}$-semigroup $\left\{e^{-\mathscr{A t}}\right\}_{t \geq 0}$.

The next result of this chapter is shown in the given theorem below.

Theorem 21. Under assumptions (A) and $\left(\mathrm{H}_{2}\right)-\left(\mathrm{H}_{4}\right)$, problem (6)-(7), which is equivalent to the systems equations (1)-(5) (TDGL equations), has a mild solution $u=u(t)$ for each $u_{0} \in \mathscr{L}^{2}$ which is globally attractive and locally uniformly attractive.

\section{References}

[1] V. L. Ginzburg and L. D. Landau, "On the theory of superconductivity," Zhurnal Eksperimental'noi i Teoreticheskoi Fiziki, vol. 20, pp. 1064-1082, 1950, English translation in: L. D. Landau, Men of Physics, D. ter Haar ed, Vol. I. Pergamon Press, Oxford, UK, pp. 138-167, 1965.

[2] S. Aizicovici and H. Lee, "Nonlinear nonlocal Cauchy problems in Banach spaces," Applied Mathematics Letters, vol. 18, no. 4, pp. 401-407, 2005.

[3] S. Aizicovici and M. McKibben, "Existence results for a class of abstract nonlocal Cauchy problems," Nonlinear Analysis. Theory, Methods \& Applications A, vol. 39, no. 5, pp. 649-668, 2000.

[4] M. McKibben, Discovering Evolution Equation with Application, CRC Press Taylor \& Francis Group, 2011.

[5] S. Aizicovici and V. Staicu, "Multivalued evolution equations with nonlocal initial conditions in Banach spaces," Nonlinear Differential Equations and Applications, vol. 14, no. 3-4, pp. 361376, 2007.

[6] R. P. Agarwal and D. O'Regan, Infinite Interval Problems for Differential, Difference and Integral Equations, Kluwer Academic Publishers, Dordrecht, The Netherlands, 2001.

[7] Z. Fan, "Existence of nondensely defined evolution equations with nonlocal conditions," Nonlinear Analysis. Theory, Methods \& Applications A, vol. 70, no. 11, pp. 3829-3836, 2009.

[8] Z. Fan, "Impulsive problems for semilinear differential equations with nonlocal conditions," Nonlinear Analysis. Theory, Methods \& Applications A, vol. 72, no. 2, pp. 1104-1109, 2010.

[9] Z. Fan and G. Li, "Existence results for semilinear differential equations with nonlocal and impulsive conditions," Journal of Functional Analysis, vol. 258, no. 5, pp. 1709-1727, 2010.
[10] J. García-Falset, "Existence results and asymptotic behavior for nonlocal abstract Cauchy problems," Journal of Mathematical Analysis and Applications, vol. 338, no. 1, pp. 639-652, 2008.

[11] J. Liang, J. H. Liu, and T.-J. Xiao, "Nonlocal impulsive problems for nonlinear differential equations in Banach spaces," Mathematical and Computer Modelling, vol. 49, no. 3-4, pp. 798-804, 2009.

[12] L. Zhu and G. Li, "On a nonlocal problem for semilinear differential equations with upper semicontinuous nonlinearities in general Banach spaces," Journal of Mathematical Analysis and Applications, vol. 341, no. 1, pp. 660-675, 2008.

[13] Q. Liu and R. Yuan, "Existence of mild solutions for semilinear evolution equations with non-local initial conditions," Nonlinear Analysis. Theory, Methods \& Applications A, vol. 71, no. 9, pp. 4177-4184, 2009.

[14] C. Xie, W. Zhong, and Z. Fan, "Existence results for nonlinear nonlocal problems in Banach spaces," Applied Mathematics Letters, vol. 22, no. 7, pp. 998-1002, 2009.

[15] T.-J. Xiao and J. Liang, "Existence of classical solutions to nonautonomous nonlocal parabolic problems," Nonlinear Analysis, Theory, Methods and Applications, vol. 63, no. 5-7, pp. e225e232, 2005.

[16] X. Xue, " $L^{p}$ theory for semilinear nonlocal problems with measure of noncompactness in separable Banach spaces," Journal of Fixed Point Theory and Applications, vol. 5, no. 1, pp. 129-144, 2009.

[17] X. Xue, "Nonlinear differential equations with nonlocal conditions in Banach spaces," Nonlinear Analysis. Theory, Methods \& Applications A, vol. 63, no. 4, pp. 575-586, 2005.

[18] X. Xue, "Existence of solutions for semilinear nonlocal Cauchy problems in Banach spaces," Electronic Journal of Differential Equations, vol. 64, p. 7, 2005.

[19] X. Xue, "Nonlocal nonlinear differential equations with a measure of noncompactness in Banach spaces," Nonlinear Analysis. Theory, Methods \& Applications A, vol. 70, no. 7, pp. 2593-2601, 2009.

[20] L. Olszowy, "Existence of mild solutions for the semilinear nonlocal problem in Banach spaces," Nonlinear Analysis. Theory, Methods \& Applications A, vol. 81, pp. 211-223, 2013.

[21] D. Bothe, "Multivalued perturbations of $m$-accretive differential inclusions," Israel Journal of Mathematics, vol. 108, pp. 109-138, 1998.

[22] H.-S. Ding, J. Liang, G. M. N’Guérékata, and T.-J. Xiao, "Mild pseudo-almost periodic solutions of nonautonomous semilinear evolution equations," Mathematical and Computer Modelling, vol. 45, no. 5-6, pp. 579-584, 2007.

[23] J. García-Falset, "The asymptotic behavior of the solutions of the Cauchy problem generated by $\varphi$-accretive operators," Journal of Mathematical Analysis and Applications, vol. 310, no. 2, pp. 594608, 2005.

[24] Z. Fan and G. Li, "Asymptotic behavior of the solutions of nonautonomous systems in Banach spaces," Nonlinear Analysis. Theory, Methods \& Applications A, vol. 68, no. 12, pp. 3733-3741, 2008.

[25] J. Banaś, "On existence theorems for differential equations in Banach spaces," Bulletin of the Australian Mathematical Society, vol. 32, no. 1, pp. 73-82, 1985.

[26] J. Banaś, "Measures of noncompactness in the space of continuous tempered functions," Demonstratio Mathematica, vol. 14, no. 1 , pp. 127-133, 1981. 
[27] J. Banaś and K. Goebel, Measures of Noncompactness in Banach Spaces, vol. 60 of Lecture Notes in Pure and Applied Mathematics, Marcel Dekker, New York, NY, USA, 1980.

[28] J. Banaś and M. Lecko, "Solvability of infinite systems of differential equations in Banach sequence spaces," Journal of Computational and Applied Mathematics, vol. 137, no. 2, pp. 363375, 2001.

[29] J. Banaś and K. Sadarangani, "Compactness conditions in the study of functional, differential, and integral equations," Abstract and Applied Analysis, vol. 2013, Article ID 819315, 14 pages, 2013.

[30] M. Mursaleen and S. A. Mohiuddine, "Applications of measures of noncompactness to the infinite system of differential equations in $\ell_{p}$ spaces," Nonlinear Analysis. Theory, Methods \& Applications A, vol. 75, no. 4, pp. 2111-2115, 2012.

[31] L. Olszowy, "Solvability of some functional integral equation," Dynamic Systems and Applications, vol. 18, no. 3-4, pp. 667-676, 2009.

[32] L. Olszowy, "On some measures of noncompactness in the Fréchet spaces of continuous functions," Nonlinear Analysis. Theory, Methods \& Applications A, vol. 72, pp. 2119-2126, 2010.

[33] L. Olszowy, "On existence of solutions of a quadratic Urysohn integral equation on an unbounded interval," Commentationes Mathematicae, vol. 48, no. 1, pp. 103-112, 2008.

[34] L. Olszowy, "Existence of mild solutions for semilinear nonlocal Cauchy problems in separable Banach spaces," Journal of Analysis and its Applications, vol. 32, no. 2, pp. 215-232, 2013.

[35] L. Olszowy, "Fixed point theorems in the Fréchet space $C\left(R_{+}\right)$ and functional integral equations on an unbounded interval," Applied Mathematics and Computation, vol. 218, no. 18, pp. 9066-9074, 2012.

[36] J. Banaś and D. O'Regan, "On existence and local attractivity of solutions of a quadratic Volterra integral equation of fractional order," Journal of Mathematical Analysis and Applications, vol. 345, no. 1, pp. 573-582, 2008.

[37] L. D. Landau, E. M. Lifshitz, and L. P. Pitaevski, Electrodynamics of Continuous Media, vol. 8, Butterworth-Heinemann, 2nd edition, 1984.

[38] L. D. Landau and E. M. Lifshitz, Fluid Mechanics, vol. b, Butterworth-Heinemann, 2nd edition, 1987.

[39] R. Temam, Infinite-Dimensional Dynamical Systems in Mechanics and Physics, vol. 68, Springer, New York, NY, USA, 2nd edition, 1997.

[40] J. K. Hale, Asymptotic Behavior of Dissipative Systems, vol. 25 of Mathematical Surveys and Monographs, American Mathematical Society, Providence, RI, USA, 1988.

[41] R. A. Adams and J. J. F. Fournier, Sobolev Spaces, vol. 140, Elsevier, 2nd edition, 2003.

[42] V. Maz'ya, Sobolev Spaces with Applications to Elliptic Partial Differential Equations, Springer, Heidelberg, Germany, 2nd edition, 2011.

[43] V. Georgescu, "Some boundary value problems for differential forms on compact Riemannian manifolds," Annali di Matematica Pura ed Applicata, vol. 122, pp. 159-198, 1979.

[44] D. Henry, Geometric Theory of Semilinear Parabolic Equations, vol. 840 of Lecture Notes in Mathematics, Springer, New York, NY, USA, 1981.

[45] V. Girault and P.-A. Raviart, Finite Element Methods for NavierStokes Equations, Springer, New York, NY, USA, 1986.

[46] J. Bardeen, L. N. Cooper, and J. R. Schrieffer, "Theory of superconductivity," Physical Review, vol. 108, pp. 1175-1204, 1957.
[47] A. A. Abrikosov, Fundamental of the Theory of Metals, NorthHolland, Amstedram, The Netherlands, 1988.

[48] M. Tinkham, Introduction To Superconductivity, McGraw-Hill, New York, NY, USA, 2nd edition, 1996.

[49] P. DeGennes, Superconductivity in Metals and Alloys, Benjamin, New York, NY, USA, 1966.

[50] H. Brezis, Functional Analysis, Sobolev Spaces and Partial Differential Equations, Springer, New York, NY, USA, 2011.

[51] E. Zeidler, Nonlinear Functional Analysis and its Applications, vol. 2, Springer, New York, NY, USA, 1990.

[52] A. Schmid, "A time dependent Ginzburg-Landau equation and its application to the problem of resistivity in the mixed state," Physik der Kondensierten Materie, vol. 5, no. 4, pp. 302-317, 1966.

[53] C. M. Elliot and Q. Tang, Existence Theorems For an Evolutionary Superconductivity Model, University of Sussex, Brighton, UK, 1992.

[54] Q. Tang, "On an evolutionary system of Ginzburg-Landau equations with fixed total magnetic flux," Communications in Partial Differential Equations, vol. 20, no. 1-2, pp. 1-36, 1995.

[55] Q. Du, "Global existence and uniqueness of solutions of the time-dependent Ginzburg-Landau model for superconductivity," Applicable Analysis, vol. 53, no. 1-2, pp. 1-18, 1994.

[56] Z. M. Chen, K.-H. Hoffmann, and J. Liang, "On a nonstationary Ginzburg-Landau superconductivity model," Mathematical Methods in the Applied Sciences, vol. 16, no. 12, pp. 855-875, 1993.

[57] J. Liang and Q. Tang, "Asymptotic behaviour of the solutions of an evolutionary Ginzburg-Landau superconductivity model," Journal of Mathematical Analysis and Applications, vol. 195, no. 1, pp. 92-107, 1995.

[58] L. P. Gor'kov and G. M. Eliasherberg, "Generalization of the Ginzburg-Landau equations for an nonstationary problems in the case of alloys with paramagnetic imparties," Zhurnal Eksperimental'noi $i$ Teoreticheskoi Fiziki, vol. 54, pp. 612-626, 1968, Soviet Physics, vol. 27, pp. 328-334, 1968.

[59] J. Fleckinger-Pellé and H. G. Kaper, "Gauges for the GinzburgLandau equations of superconductivity," Zeitschrift fur Angewandte Mathematik und Mechanik, vol. 76, no. 2, pp. 345-348, 1996.

[60] P. Takac, "On the dynamical process generated by superconductivity model," Zeitschrift fur Angewandte Mathematik und Mechanik, vol. 76, no. 52, pp. 349-352, 1995.

[61] W. D. Gropp, H. G. Kaper, D. M. Levine, M. Palumbo, and V. M. Vinokur, "Numerical simulation of vortex dynamics in type-II superconductors," Journal of Computational Physics, vol. 123, no. 2, pp. 254-266, 1996.

[62] D. W. Braun, G. W. Crabtree, H. G. Kaper et al., "Structure of a moving vortex lattice," Physical Review Letters, vol. 76, no. 5, pp. 831-834, 1996.

[63] G. W. Crabtree, G. K. Leaf, H. G. Kaper et al., "Timedependent Ginzburg-Landau simulations of vortex guidance by twin boundaries," Physica C, vol. 263, no. 1-4, pp. 401-408, 1996.

[64] G. W. Carabtree, G. K. Leaf, H. G. Kaper, D. W. Braun, V. M. Vinokur, and A. E. Koshelev, "Dynamics vortex phases in superconductors with correlated disorder," Preprint ANL/MCSP590-0496, Mathematics and Computer Science Division, Aragonne National Laboratory, 1996.

[65] Th. Gallay, "A center-stable manifold theorem for differential equations in Banach spaces," Communications in Mathematical Physics, vol. 152, no. 2, pp. 249-268, 1993.

[66] J.-M. Ghidaglia and B. Héron, "Dimension of the attractors associated to the Ginzburg-Landau partial differential equation," Physica D, vol. 28, no. 3, pp. 282-304, 1987. 
[67] P. Mattila, Geometry of Sets and Measures in Euclidean Spaces, vol. 44, Cambridge University Press, Cambridge, UK, 1995.

[68] L. Hormander, The Analysis of Linear Partial Differential Operators, vol. 1, Springer, Berlin, Germany, 2nd edition, 1990.

[69] H. Amann, Linear and Quasilinear Parabolic Problem, Bikhauser, Basel, Switzerland, 1995.

[70] H.-P. Heinz, "On the behaviour of measures of noncompactness with respect to differentiation and integration of vector-valued functions," Nonlinear Analysis. Theory, Methods \& Applications A, vol. 7, no. 12, pp. 1351-1371, 1983.

[71] R. P. Agarwal, M. Meehan, and D. O'Regan, Fixed Point Theory and Applications, vol. 141 of Cambridge Tracts in Mathematics, Cambridge University Press, Cambridge, UK, 2001.

[72] A. Pazy, Semigroups of Linear Operators and Applications to Partial Differential Equations, Springer, New York, NY, USA, 1983.

[73] J. Fleckinger-Pellé, H. G. Kaper, and P. Takáč, "Dynamics of the Ginzburg-Landau equations of superconductivity," Nonlinear Analysis. Theory, Methods \& Applications A, vol. 32, no. 5, pp. 647-665, 1998.

[74] H. G. Kaper and P. Takáč, "An equivalence relation for the Ginzburg-Landau equations of superconductivity," Journal of Applied Mathematics and Physics, vol. 48, no. 4, pp. 665-675, 1997. 


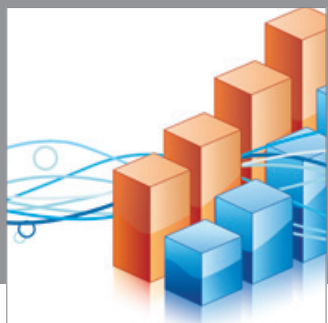

Advances in

Operations Research

mansans

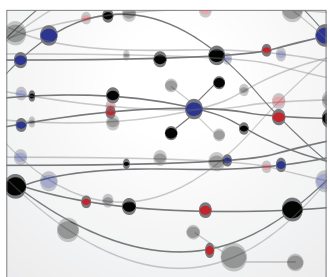

The Scientific World Journal
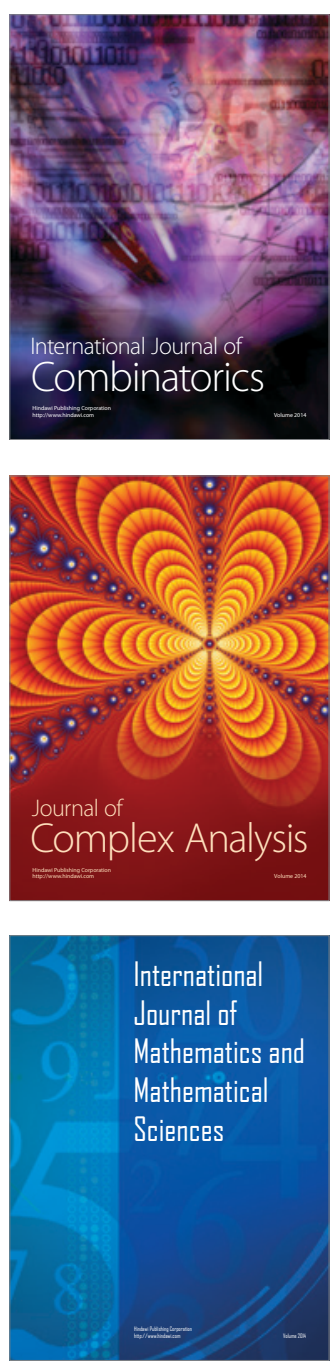
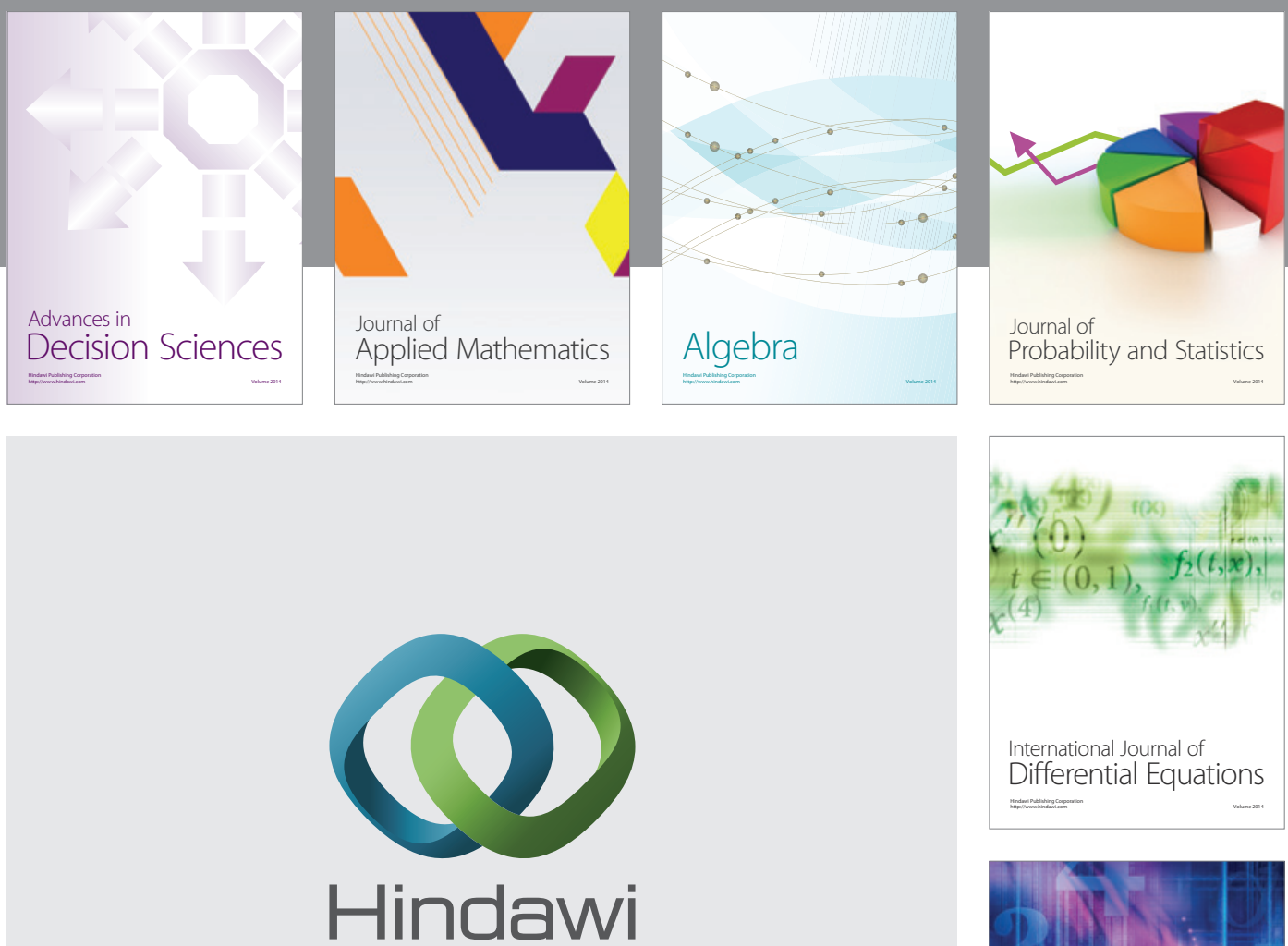

Submit your manuscripts at http://www.hindawi.com
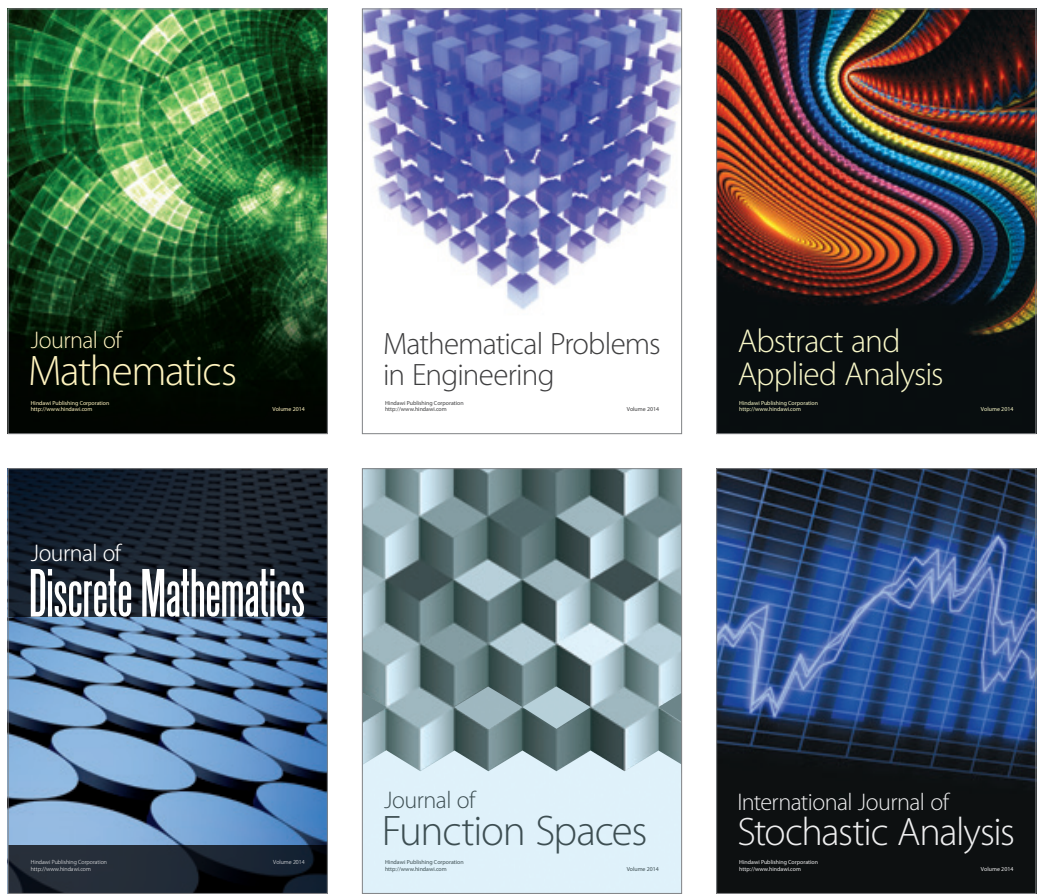

Journal of

Function Spaces

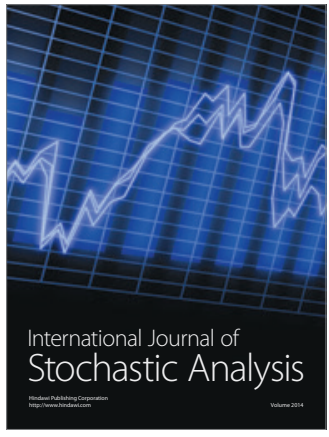

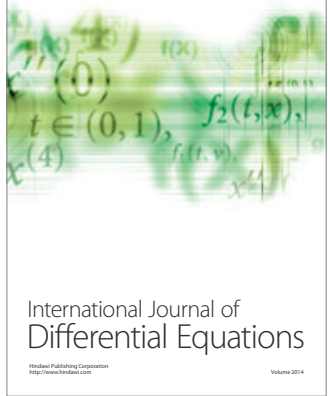
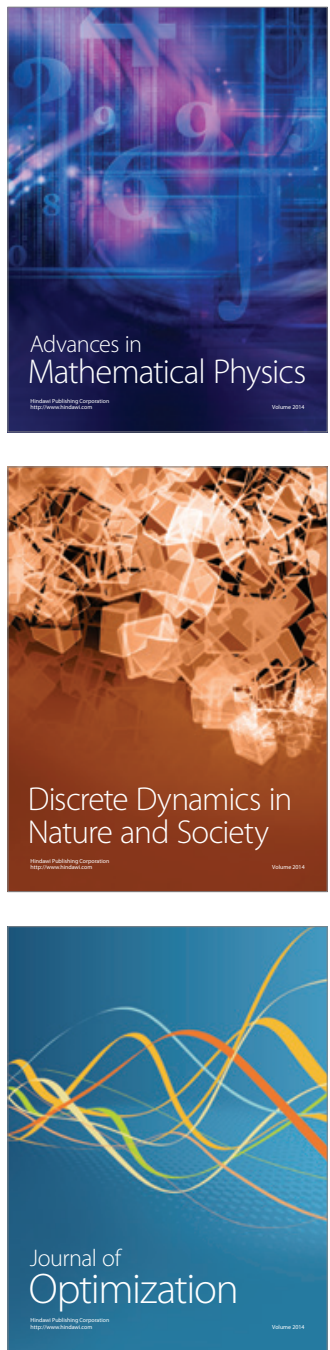